\title{
Stability of FDTD in Curvilinear Coordinates
}

\author{
Lukas Pauk, student member, Zbynek Skvor, senior member IEEE \\ Department of Electromagnetic Field, \\ Czech Technical University in Prague, \\ Technicka 2, 16627 Praha 6, Czech Republic, \\ paukl@fel.cvut.cz,skvor@fel.cvut.cz, Phone: +420-2-24352276, fax:+420-2-3119958
}

\begin{abstract}
A new approach suitable for determination of the maximal stable time step for the finite-difference timedomain (FDTD) algorithm in curvilinear coordinates is presented. It is based on a modified variable separation method, applied to the set of difference equations of the FDTD algorithm. Investigation is carried out in spherical and cylindrical coordinates. A simple yet accurate enough approximative formula for cylindrical coordinates is presented. Applied to Cartesian coordinates, this approach yields the well-known Courrant condition.
\end{abstract}

\section{Introduction}

The solution of Maxwell's equations in the time domain is becoming increasingly important as a tool for analyzing microwave components and systems. The precision of modeling of electromagnetic field is a tricky question. One major factor that has a considerable influence on the precision is the time step of the FDTD algorithm. An optimal value of the time step exists, resulting in fastest and most precise computation. The other reason one should set this constant properly is that even a small excess over its optimal value would result in instability of the algorithm. There is a condition for the time step called Courant ([1]), valid only for rectangular coordinates. We used a new approach to find the best time step that is not restricted to rectangular coordinates.

\section{Method}

\subsection{Introduction - description of the algorithm}

First, let us describe the form that we will designate the derivative:

$$
A, x^{2} \quad \text { let be the same as } \quad \frac{\partial^{2}}{\partial x^{2}} A .
$$

Using this symbolic, let us write first two Maxwell's equations in general curvilinear coordinates $\left(u_{1}, u_{2}, u_{3}\right)$ :

$$
\begin{aligned}
& \frac{+\varepsilon}{e^{\frac{\sigma_{E}}{\varepsilon}}}\left(e^{\frac{\sigma_{E}}{\varepsilon} t} E_{u_{1}}\right), t=\frac{1}{u_{2} u_{3}}\left[\left(u_{3} H_{u_{3}}\right), u_{2}-\left(u_{2} H_{u_{2}}\right), u_{3}\right] \\
& \frac{-\mu}{e^{\frac{\sigma_{M}}{\mu} t}}\left(e^{\frac{\sigma_{M}}{\mu} t} H_{u_{1}}\right), t=\frac{1}{u_{2} u_{3}}\left[\left(u_{3} E_{u_{3}}\right), u_{2}-\left(u_{2} E_{u_{2}}\right), u_{3}\right]
\end{aligned}
$$

where the curl operators are written by means of local length units $\left(h_{1}, h_{2}, h_{3}\right)$ in the coordinate system (e.g. [2]). (The missing $2 \times 2$ equations are formed by cyclic replacement of indices 1,2,3.)

The FDTD algorithm we are interested in is the commonly used first order leapfrog one. The discretization mesh ([3],[4]) is designed so that along each coordinate axis the samples of components of vector $\mathrm{E}$ and $\mathrm{H}$ were interlaced.

Our equations are formed of terms of the following form:

$$
\frac{1}{f}(f C), x
$$

where $C$ is a component of the vector and $x$ is a coordinate $\left(u_{1}, u_{2}, u_{3}, t\right)$. For FDTD we need to approximate this term using samples of $C$. We will use only two neighboring samples (first order FDTD). The place where the approximation is demanded to be most precise is in the center between the samples (it follows from the formation of the mesh). This is the leapfrog principle, which is most convenient.

The possible solution of the problem can be:

$$
\frac{1}{f}(f C), x \approx \frac{1}{\operatorname{Av}\left(f,\left\langle x_{0}, x_{1}\right\rangle\right)} \cdot \frac{f\left(x_{1}\right) C_{1}-f\left(x_{0}\right) C_{0}}{\Delta x}
$$

where $\operatorname{Av}\left(f,\left\langle x_{0}, x_{1}\right\rangle\right)=\frac{1}{\Delta x} \int_{x_{0}}^{x_{1}} f d t, \Delta x=x_{1}-x_{0}$

$, C_{0}, C_{1}$ are the samples, which are located at positions $x_{0}, x_{1}$, (other coordinates remain constant), the result is most precise at $x_{0}+\frac{1}{2} \Delta x$.

If we introduce this discretization to the equations (1), we obtain the FDTD algorithm; the form in rectangular coordinates with lossy media can be found e.g. in [1], the one in cylindrical coordinates in [4].

We will not substitute this in (1) using lots of confusing indices, but let us write the algorithm exactly the same way as (1). Before operating with these 
equations, however, we will have to check whether the operation remains correct even with the discretized form.

\subsection{Method}

The method we used to determine the time step is based on modified variable separation method, applied to the set of difference equations (1). We will assume each component of the field vectors to be a product of 4 functions that each depends on only one coordinate:

$$
\begin{gathered}
C_{x}=C_{x}^{u_{1}}\left(u_{1}\right) C_{x}^{u_{2}}\left(u_{2}\right) C_{x}^{u_{3}}\left(u_{3}\right) C_{x}^{t}(t) \\
C \ldots E, H ; x \ldots u_{1}, u_{2}, u_{3}, t
\end{gathered}
$$

A separate set of difference equations for each part of $C_{x}$ can be obtained and the coefficients in the set do not depend on the other coordinates as well.

The difference equations for time (lossless medium for simplicity, leaving out upper indices) are:

$$
E, t=\frac{1}{\varepsilon} k H, H, t=-\frac{1}{\mu} k E
$$

where $E=E_{x}, H=H_{x}, x \ldots u_{1}, u_{2}, u_{3}$

$k$ is a constant, which connects these equations with the remaining sets.

On the contrary to the continuous case, the solution for its discretized counterpart may diverge unless the following condition is fulfilled:

$$
k<\frac{2 \sqrt{\mu \varepsilon}}{\Delta t}
$$

This is the principle of FDTD instability.

To be able to apply the variable separation method, we investigated FDTD stability in a mesh with the boundaries complying with the coordinate system. The boundaries that enclose the mesh are given by:

$$
u_{i}=\text { const }=U_{i 1}, u_{i}=\text { const }=U_{i 2} ; i \ldots 1,2,3
$$

Boundary condition has been applied, which remained the same on these separate parts of boundaries.

In this case it is possible to determine the unknown constant $k$ (4) by solving the remaining ordinary (nonpartial - involving only one variable) difference equation sets with the corresponding boundary conditions at $U_{i 1}, U_{i 2}$. These constants correspond to characteristic functions - let us call them mesh modes. The number of solutions is not infinite, as it was in the continuous case. To ensure stability of the sum of all the possible modes, we must fulfill condition (5) for all the mode constants. Only the largest constant is of interest as it yields to the smallest time step:

$$
\Delta t<\frac{2 \sqrt{\mu \varepsilon}}{k_{\max }}
$$

Finally it should be noted that a linear combination of the mesh modes can make up arbitrary initial state of the mesh. In other words, using variable separation method (3) does not leave out any solution that could be expressed in the mesh.

\section{Results}

The mesh of all the cases we investigated had the simplest termination - condition that modeled ideal metallic boundary - components of vector $E$ tangential to the surface were set to zero.

The results obtained were verified by means of the FDTD algorithm itself. We have experimentally verified the time step resulting from our analysis in the following manner: We made two experiments for each case. One experiment has been carried out with time step slightly over our predicted value, and it proved to diverge in all cases. Second experiment has been made with time-step slightly under our predicted value, and it proved to be stable in all cases. By "slightly" we mean here a relative change of one part per billion.

For convenience, all the numerical results in the following text will be normalized to:

$$
\varepsilon=\mu=1
$$

\subsection{Rectangular coordinates $(x, y, z)$}

The equation sets are the same for all the space coordinates, we will write them only for dimension $x$ :

$$
E_{y}, x=k_{x} H_{z}, H_{z}, x=-k_{x} E_{x}, \ldots
$$

The demanded constant $k$ is composed of the partial constants in the following way:

$$
k^{2}=k_{x}^{2}+k_{y}^{2}+k_{z}^{2}
$$

For the specified boundary condition they can be be solved analytically, the formula for the largest constant is this one:

$$
\left(k_{x} \Delta x\right)^{2}=2+2 \cos \left(\frac{\pi}{N_{x}}\right)
$$

where $N_{x}$ is the number of $\Delta x$ in the mesh (number of cells between the zero boundary condition).

The resulting time step limit is thus following:

$$
\frac{\Delta t}{\sqrt{\mu \varepsilon}}<\left(\frac{\frac{1}{2}+\frac{1}{2} \cos \left(\pi / N_{x}\right)}{\Delta x^{2}}+\frac{\frac{1}{2}+\frac{1}{2} \cos \left(\pi / N_{y}\right)}{\Delta y^{2}}+\ldots\right)^{-\frac{1}{2}}
$$

The time step limit is a bit higher than that obtained by the Currant condition ([1]), but for meshes that are dense enough, it is practically the same. 


\subsection{Cylindrical coordinates $(r, \varphi, z)$}

The situation with this coordinate system is a bit more complex. The equations for the $z$ and $\varphi$ are virtually the same as those for the rectangular system. There is absolutely no problem to cancel out the field dependence along $z$ or $\varphi$ (the case presented in [1]) coordinate (or both at the same time). For sake of simplicity we did so for $z$ and obtained FDTD in two dimensions only. Then we confined to the mesh modes with $k_{z}=0$.

The only equation set that must be solved numerically is the one for $r$ coordinate (again, leaving out upper indices):

$$
\begin{gathered}
\frac{1}{r}\left(r H_{\varphi}\right), r=k E_{z}\left[1-\frac{1}{r^{2}} \cdot \frac{k_{\varphi}^{2}}{k^{2}}\right] \\
E_{z}, r=-k H_{\varphi}
\end{gathered}
$$

After eliminating a component we would arrive to the Bessel differential equation. In the continuous case we could use roots of Bessel functions to determine the constant $k$, but in our case we have to solve the difference equation again. It can be shown that it is the largest $k_{\varphi}$ that would yield the largest $k$, which we are to find. We can get the largest $k_{\varphi}$ the same way as we did in the rectangular case, as the equations are the same.

Because cylindrical coordinates are used quite often, we found a simple approximative formula for the $k$. There was also one significant requirement to the approximation - the time step computed according to it (by means of (6)) had to be smaller or equal to the critical one. Here it is:

$$
k^{2}=\frac{2}{\Delta r^{2}}+\frac{1}{\Delta r^{2}} \sqrt{4-\frac{\Delta r}{R_{1}+\Delta r}}+\frac{k_{\varphi}^{2}}{\left(R_{1}+\Delta r\right)^{2}}
$$

where $R_{1}$ is the smaller radius of the vector component that the zero boundary condition is applied to, $\Delta r$ is the cell dimension in the direction of $r$.
Following figures catch some interesting dependencies:

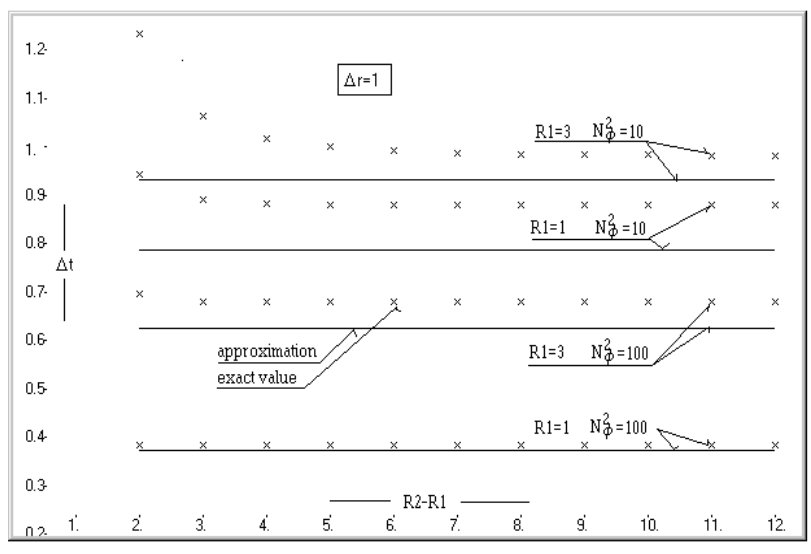

Fig. 1. Dependence of the exact time step and its approximation on the larger radius.

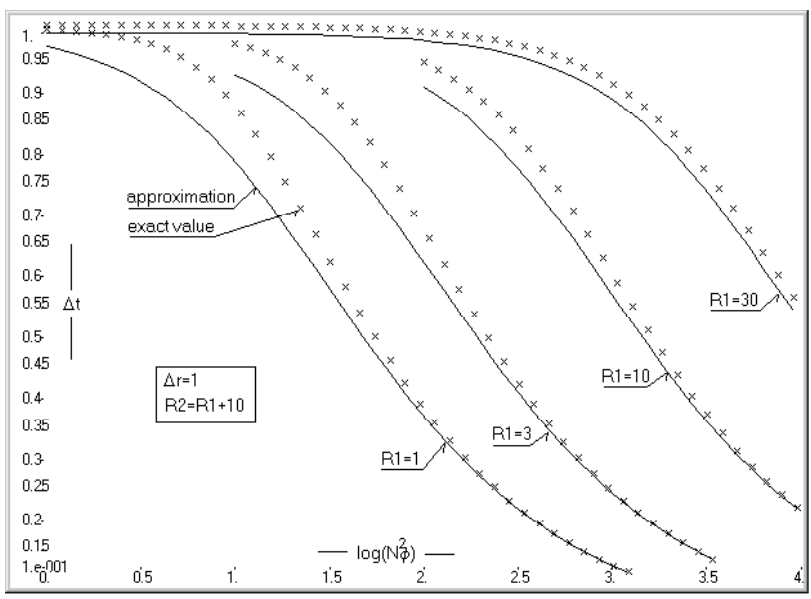

Fig. 2. Dependence of the exact time step and its approximation on the $\varphi$-coordinate characteristic constant $\left(\mathrm{k}_{\varphi}\right)$.

The conclusions follow:

The time step does not depend much on the larger radius $R_{2}$, if $\Delta r$ remains constant. The value stabilizes very quickly and for practical mesh dimensions varies in a negligible way. The approximation is quite good for large constant $k_{\varphi}\left(k_{\varphi}^{2}=10000\right.$ and more), which is also fulfilled in practical case. 


\subsection{Spherical coordinates $(r, \vartheta, \varphi)$}

In this case there is again a coordinate, that can be managed easily. It is the $\varphi$ one. But the remaining two are a bit worse to handle. We proceeded with full generality. The only exception is that the FDTD algorithm under investigation was again a 2 dimensional one, as the $\varphi$ variable does not bring any problem at all.

The separated difference equation sets are following: - for variable $r$ :

$$
\begin{gathered}
\frac{1}{r}\left(r H_{\varphi}\right), r=k E_{\vartheta}\left[1-\frac{1}{r^{2}} \cdot \frac{k_{\vartheta}^{2}}{k^{2}}\right] \\
\frac{1}{r}\left(r E_{\vartheta}\right), r=-k H_{\varphi} \\
E_{r}=0, E_{\vartheta}=E_{\varphi}, H_{\vartheta}=H_{\varphi}
\end{gathered}
$$

- for variable $\vartheta$ :

$$
\begin{aligned}
\frac{1}{\sin (\vartheta)}\left[\sin (\vartheta) E_{\varphi}\right], \vartheta & =k_{\vartheta} H_{r}\left[1-\frac{1}{\sin ^{2}(\vartheta)} \cdot \frac{k_{\varphi}^{2}}{k_{\vartheta}^{2}}\right] \\
H_{r}, \vartheta & =-k_{\vartheta} E_{\varphi}
\end{aligned}
$$

The important thing is that one component of a field vector is zero $\left(E_{r}=0\right)$. It means that the mesh modes based on these equations could not form any field configuration and the method would not be justified.

Fortunately, it is really only a part of the modes. The rest is described by the same equations where vectors $E$ and $H$ are interchanged. The mode constants computed according these two equation sets are slightly different. We cannot say in advance, which of the sets would yield the largest one.

At this place let us show here one concrete result for this coordination system. The boundary conditions were at:

$$
\begin{array}{ccc}
R_{1}=10, & R_{2}=16, & \Delta r=1 \\
\Theta_{1}=0.3, & \Theta_{2}=1.3, & \Delta \vartheta=\frac{1}{7} \\
k_{\varphi}^{2}=5 &
\end{array}
$$

The operator Av (2) was defined in a slightly different way:

$$
\operatorname{Av}\left(f,\left\langle x_{0}, x_{1}\right\rangle\right)=f\left(x_{0}+\frac{1}{2} \Delta x\right)
$$

It was mode $E_{r}=0$, the constant of which was the largest one, and the corresponding critical time step was:

$$
0.71575420415 \pm
$$

$\pm 0.00000000005$

\section{Conclusion}

A method, capable of predicting critical time steps in curvilinear coordinates, has been presented. The method yields results with extreme precision. It is applicable to certain boundary conditions and mesh shapes, which were not handled by approaches published so far. In addition, a simple approximative formula useful in cylindrical meshes has been presented.

\section{Acknowledgement}

This work has been jointly supported by research program "Telecommunication and Information Technology" No.: J04/98:210000015 of the Czech Ministry of Education and the Grant Agency of Czech Republic program "Time-Domain Microwave Circuit Analysis", No. 102/01/571.

\section{References}

[1] A. Taflove, "Computational electrodynamics - the finite-difference time-domain method" Artech House, Boston, London, 1995.

[2] A. Angot, "Complements de mathematique a l'usage des ingenieurs de l'electrotechnique et des telecomunications", MASSON et Cie (Editions de la Reuve d'Optique, Paris, 1952

[3] K. S. Yee, "Numerical solution of initial boundary value problems involving Maxwell's equations in isotropic media," IEEE Trans. Antenna Propagat., vol. AP-14, May 1966.

[4] Y. Chen, R. Mitra, P. Harms, "Finite-difference time-domain algorithm for solving Maxwell's equations in rotationally symmetric geometries" IEEE Trans. on MTT, Vol. 44, No. 6, June 1996. 